Research Paper

\title{
Identification of a novel de novo ANK1 R1426* nonsense mutation in a Chinese family with hereditary spherocytosis by NGS
}

\author{
Xiong Wang ${ }^{1, *}$, Bin Yii,*, Ketao Mu ${ }^{3}$, Na Shen ${ }^{1}$, Yaowu Zhu ${ }^{1}$, Qun Hu${ }^{4}$ and Yanjun Lu ${ }^{1}$ \\ ${ }^{1}$ Department of Laboratory Medicine, Tongji Hospital, Tongji Medical College, Huazhong University of Science and Technology, \\ Wuhan 430030, China \\ ${ }^{2}$ Department of Pediatric Surgery, Tongji Hospital, Tongji Medical College, Huazhong University of Science and Technology, \\ Wuhan 430030, China \\ ${ }^{3}$ Department of Radiology, Tongji Hospital, Tongji Medical College, Huazhong University of Science and Technology, Wuhan \\ 430030, China \\ ${ }^{4}$ Department of Pediatrics, Tongji Hospital, Tongji Medical College, Huazhong University of Science and Technology, Wuhan \\ 430030, China \\ *These authors contributed equally to this work
}

Correspondence to: Qun Hu, email: qunhu2010@163.com

Yanjun Lu, email: junyanlu_2000@163.com

Keywords: hereditary spherocytosis, splenectomy, anemia, ankyrin, mutation

Received: April 14, 2017

Accepted: May 15, 2017

Published: May 27, 2017

Copyright: Wang et al. This is an open-access article distributed under the terms of the Creative Commons Attribution License 3.0 (CC BY 3.0), which permits unrestricted use, distribution, and reproduction in any medium, provided the original author and source are credited.

\section{ABSTRACT}

Hereditary spherocytosis (HS) is an inherited heterogeneous hemolytic anemia, characterized by the presence of spherical-shaped erythrocytes on the peripheral blood smear, and the clinical manifestation ranges from asymptomatic to severely anemic, and transfusion-dependent patients. Mutations in at least five genes (ANK1, EPB42, SLC4A1, SPTA1, and SPTB) have been identified so far, and mutations of ANK1 gene are responsible for the majority of all HS cases. In this study, targeted next generation sequencing (NGS) was applied to identify a novel de novo ANK1 c.4276C>T (p.R1426*) nonsense mutation in a Chinese family with a patient of HS who was diagnosed clinically with only $10 \%$ spherical-shaped erythrocytes in the peripheral blood and received splenectomy. Sanger sequencing further confirmed that only the patient carried heterozygous ANK1 c.4276C $>$ T nonsense mutation, while none of his parents or his young brother carried this mutation. Moreover, consistent with the genetic findings, the anemia was ameliorated after splenectomy. RBCs increased from $2.74 \times 1012 / L$ pre-surgery to $4.76 \times 1012 / L$ one month post-surgery, and hemoglobin increased from $66 \mathrm{~g} / \mathrm{L}$ to $126 \mathrm{~g} / \mathrm{L}$ respectively. This is the first report of ANK1 c.4276C>T (p.R1426*) heterozygous nonsense mutation responsible for HS. Our results also demonstrate that targeted NGS may provide a powerful approach for rapid genetic test of HS.

\section{INTRODUCTION}

Hereditary spherocytosis (HS) is the most frequent form of inherited hemolytic anemia characterized by the presence of spherical-shaped erythrocytes on the peripheral blood smear, hemolysis, splenomegaly, jaundice, and gallstones [1]. The clinical severity of HS varied widely, and its prevalence is 1 in 2,000 in Northern European population, and the prevalence in China is about
1 in 100,000 people [2]. To date, mutations in at least five genes (ANK1, EPB 42, SLC4A1, SPTA1, and SPTB), encoding ankyrin, protein 4.2 , band 3 protein, $\alpha$-spectrin, and $\beta$-spectrin respectively, are associated with HS.

The majority of HS cases is associated with autosomal dominant (AD) pattern, although autosomal recessive (AR) inheritance has also been described. Mutations in ANK1 (mainly nonsense and frame shift mutations) are responsible for about $75 \%$ of cases of HS 
in humans, followed by mutations in SLC4A1 and SPTB genes $[3,4]$. Ankyrin typically consists of three structural domains: a multiple ankyrin repeats $\mathrm{N}$-terminal domain, a spectrin-binding center region, and a regulatory $\mathrm{C}$-terminal domain, which is the least conserved $[5,6]$. Erythroid ankyrin is a major protein in RBCs involved in the linkage of transmembrane proteins and the cell membrane skeleton via spectrin, band 4.2 protein, band 3 protein, and ankyrin deficiency results in reduced incorporation of spectrin [7]. Up to now, 59 distinct $A N K 1$ mutations have been reported in human HS in HGMD database (www. hgmd.cf.ac.uk), including deletion, frameshift, nonsense, or missense mutations.

Recent advances in next generation sequencing (NGS) approach provide rapid detection of genetic mutations, especially for large panel including a variety of candidate genes, leading the field of genetic testing away from Sanger sequencing [8]. In this study, we analyzed the disease-causing mutations in a Chinese family with a patient of HS using targeted NGS to identify the mutational characteristics in ANK1, EPB42, SLC4A1, $S P T A 1$, and $S P T B$ genes, followed by Sanger sequencing confirmation.

In this study, a novel de novo $A N K 1$ c. $4276 \mathrm{C}>\mathrm{T}$ (p.R1426*) heterozygous nonsense mutation in the patient characterized with only $10 \%$ spherical-shaped erythrocytes in the peripheral blood, anemia, and splenomegaly, while none of his parents or his young brother carried this mutation. Moreover, consistent with the genetic findings, the anemia was ameliorated after splenectomy. This study also demonstrates that targeted NGS is an effective method for identifying causal mutations for HS diagnosis.

\section{RESULTS}

\section{Clinical features of a chinese family with HS}

An 8 years old male patient with anemia, jaundice, and splenomegaly, received splenectomy due to severe anemia although only $10 \%$ spherical-shaped erythrocytes on the peripheral blood smear. The family tree was drawn (Figure 1A), and the splenomegaly identified by CT scanning was shown in Figure 1B. The changes of RBC and Hemoglobin of the patient with HS pre/post-surgery was recorded in Table 1, and the anemia was ameliorated after splenectomy.

\section{NGS output and coverage}

The sequencing of coding exons and adjcant intronic regions of $5 \mathrm{HS}$-associated genes on the Ion torrent PGM achieved an average output of 463,333 mapped reads and $98.83 \%$ on target. In summary, $100 \%$ of all target amplicons was covered at least once, $99.57 \%$ amplicons was covered at least 20 times, 97.85\% amplicons was covered at least 100 times. The mean uniformity of base coverage is $94.50 \%$ in this panel. The average read depth was 1745 folds (Figure 2A).

\section{Mutation detection and sanger sequencing validation}

NGS identified a total of 40 variants in the patient subjected to a process to discover pathogenic mutations (Supplementary Table 1). After applying filtration process and annotation, a heterozygous $A N K 1$ c.4276C $>\mathrm{T}$ (p.R1426*) nonsense mutation was identified which could be implicated in the patient's phenotype.

Sanger sequencing of this loci was performed on all immediate family members. The result showed that only the patient carried this mutation, while none of his parents or his young brother held this mutation (Figure 2B). Paternity testing by haplotype analysis confirmed that these were the biological parents of the patient with HS (Table 2). These data indicate a de novo mutation.

Moreover, ANK1 c. $4276 \mathrm{C}>\mathrm{T}$ mutation (hg19: chr8:41546059G $>$ A) was not found in RBCmembrane, 1000G, ExAC, HGMD, or EVS database.
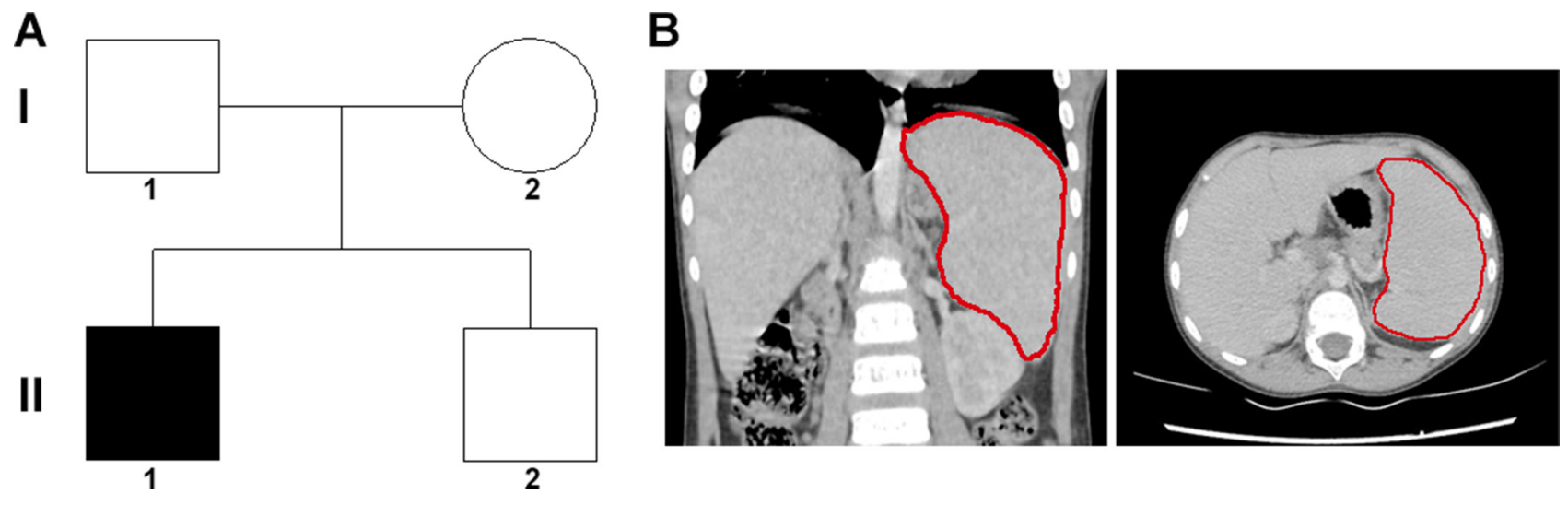

Figure 1: Family tree and clinical characteristics of the patient. (A) the family tree of a Chinese family with patient of HS. A filled square was patient, and unfilled square or circle denoted unaffected male or female respectively. (B) the re-constructed CT scanning image, and the red line outlined the spleen. 
Table 1: RBC and Hemoglobin changes of the patient with HS

\begin{tabular}{ccccc}
\hline Test & $\begin{array}{c}\text { 3 Months Pre- } \\
\text { operation }\end{array}$ & $\begin{array}{c}\text { 1 DayPre- } \\
\text { operation }\end{array}$ & $\begin{array}{c}\text { 1 Week Post- } \\
\text { operation }\end{array}$ & $\begin{array}{c}\text { 1 Month Post- } \\
\text { operation }\end{array}$ \\
\hline $\mathrm{RBC}\left(\times 10^{12} / \mathrm{L}\right)$ & 2.52 & 2.74 & 4.52 & 4.76 \\
Hemoglobin $(\mathrm{g} / \mathrm{L})$ & 58.0 & 66.0 & 118.0 & 126.0 \\
\hline
\end{tabular}

Table 2: Four SNPs selected for paternity testing

\begin{tabular}{ccccc}
\hline SNP & I-1 (Father) & I-2 (Mother) & II-1 (Proband) & II-2 (Young brother) \\
\hline rs2278621 & CT & CC & CT & CC \\
rs504574 & CG & CC & CG & CG \\
rs7826127 & CT & TT & CT & TT \\
rs1872877 & AG & AG & GG & GG \\
\hline
\end{tabular}

\section{Genetic and phenotype association analysis}

Genetic tests showed a de novo ANK1 c. $4276 \mathrm{C}>\mathrm{T}$ (p.R1426*) nonsense mutation in this family, and only the patient carried this mutation. Hemolysis, splenomegaly, and jaundice were observed only in the patient. Moreover, consistent with the genetic findings, the anemia was ameliorated after splenectomy, a reserved surgery for the treatment of HS patients with severe symptoms. RBCs increased from $2.74 \times 10^{12} / \mathrm{L}$ pre-surgery to $4.76 \times 10^{12} / \mathrm{L}$ one month post-surgery, and hemoglobin increased from $66 \mathrm{~g} / \mathrm{L}$ to $126 \mathrm{~g} / \mathrm{L}$ respectively (Table 3 ).

\section{Functional prediction of the mutation}

Ankrin includes an $89 \mathrm{kDa}$ domain in N-terminal, a UPA domain in the center, and a $55 \mathrm{kDa}$ regulatory domain in $\mathrm{C}$-terminal which is involved in regulating binding of $\beta$-spectrin and band 3 protein. ANK1 c. $4276 \mathrm{C}>\mathrm{T}$ nonsense mutation produced a short protein (p.R1426*) located in the $55 \mathrm{kDa}$ regulatory domain in C-terminal and resulted in premature termination of ankyrin.
Two kinds of software were applied to predict the effect of this mutation. ANK1 c. $4276 \mathrm{C}>\mathrm{T}$ nonsense mutation was predicted as 'disease causing' in MutationTaster, and 'Pathogenic' in InterVar with evidences of PVS1, PS2, and PM2 [9].

\section{DISCUSSION}

ANK1 mutation is the most common cause of HS, followed by mutations in SPTB, SPTA1, SLC4A1, and EPB42 ( $20 \%, \sim 5 \%, \sim 15 \%$, and $\sim 10 \%$ respectively) [10]. ANK1 mutation is inherited in both $\mathrm{AD}$ and AR patterns in HS and the majority is de novo mutation [11]. To date, 7 missense mutations, 15 nonsense mutations, 7 splicing mutations, 3 regulatory mutations, 19 small deletions, 5 small insertions, 1 small indel, 1 gross deletion, 1 gross insertion/duplication have been identified among patients with HS in HGMD database. 7 of the 15 nonsense mutations, accounting for about half of all nonsense mutations, were located in the $55 \mathrm{kDa}$ regulatory domain in C-terminal of ankrin which modulates the affinities
A

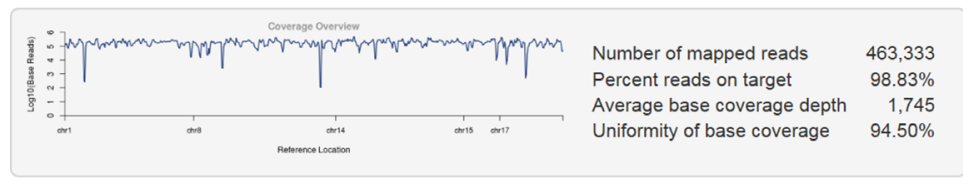

\begin{tabular}{lrllr}
\multicolumn{1}{c}{ Amplicon Read Coverage } & \multicolumn{2}{c}{ Target Base Coverage } \\
Number of amplicons & 233 & Bases in target regions & 64,768 \\
Percent assigned amplicon reads & $98.83 \%$ & Percent base reads on target & $97.32 \%$ \\
Average reads per amplicon & 1,965 & Average base coverage depth & 1,745 \\
Uniformity of amplicon coverage & $95.28 \%$ & Uniformity of base coverage & $94.50 \%$ \\
Amplicons with at least 1 read & $100.00 \%$ & Target base coverage at 1x & $100.00 \%$ \\
Amplicons with at least 20 reads & $99.57 \%$ & Target base coverage at 20x & $98.32 \%$ \\
Amplicons with at least 100 reads & $97.85 \%$ & Target base coverage at 100x & $97.26 \%$ \\
Amplicons with at least 500 reads & $94.85 \%$ & Target base coverage at 500x & $93.12 \%$ \\
Amplicons with no strand bias & $96.57 \%$ & Target bases with no strand bias & $85.04 \%$ \\
Amplicons reading end-to-end & $58.80 \%$ & Percent end-to-end reads & $71.48 \%$
\end{tabular}

B

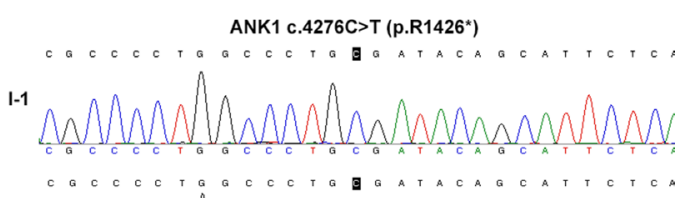

$1-2$

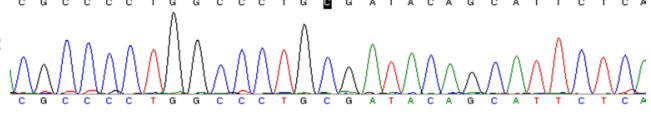

C A C C C C T G G C C C T G I G A TACA A CATTCTC

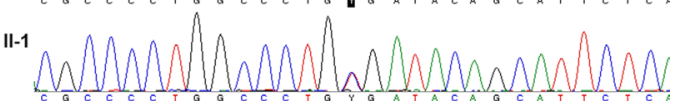

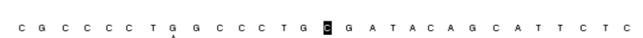

II-2

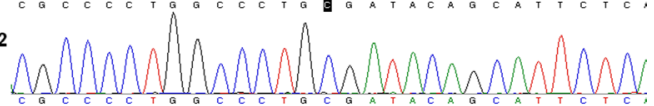

Figure 2: NGS output and mutation analysis. (A) The coverage summary of targeted NGS for HS genetic diagnosis. (B) ANK1 c. $4276 \mathrm{C}>\mathrm{T}$ (p.R1426*) mutation analysis. 
of the other domains and contains a death domain[6]. The regulatory domain modifies the association of ankyrin with the band 3 protein complex and the RBC cytoskeleton network [12]. Deficient ankyrin due to nonsense mutations in the regulatory region have been proposed as causes of HS [13]. Hughes MR et al. found that mice with truncation mutation of ankyrin lacking the spectrin-binding and regulatory C-terminal domains modeled severe hemolytic in vivo [14]. Some other proteins may also contribute to red cell skeleton, including the Rh complex, e.g. CD47 which interacts with protein 4.2 [15].

In our study, hemolysis and jaundice were found in the patient, and splenomegaly was identified by CT scanning. Genetic tests showed a de novo ANK1 c. $4276 \mathrm{C}>\mathrm{T}$ (p.R1426*) nonsense mutation in the patient, while none of his patients or his young brother carried this mutation. ANK1 c. $4276 \mathrm{C}>\mathrm{T}$ mutation was novel, and was not found in RBCmembrane, 1000G, ExAC, HGMD, or EVS database. This mutation was located in the regulatory domain in C-terminal, which might cause HS. Moreover, consistent with the genetic diagnosis, the anemia was ameliorated after splenectomy, further supporting the genotype-phenotype relationship. Our results expanded the mutation spectrum of $A N K 1$ in HS.

High-throughput NGS has promoted DNA sequencing by allowing the cost-effective and simultaneous rapid detection of multiple genes, especially in cases where traditional laboratory tests failed, or when the patients were extensively transfused [16]. Agarwal AM et al. have designed a panel including 28 genes which encode both RBC cytoskeletal proteins and enzymes to perform genetic test for hereditary haemolytic anemia [17]. Han JH et al. performed whole exome sequencing (WES) to identify casual mutation in a Korean family with HS patient [13]. We have successfully used a panel covering the complete coding region, splice site junctions, and UTR regions of the previously reported five HS related genes (OMIM\#182900, \#616649, \#270970, \#612653, and \#612690) to determine the molecular basis of patients with HS on Ion torrent PGM platform. These studies demonstrate the clinical utility of WES or targeted NGS could provide rapid genetic diagnosis for patients with inherited haemolytic anemia.

In summary, a novel de novo ANK1 c.4276C $>\mathrm{T}$ (p.R1426*) nonsense mutation was identified in a Chinese family affected by HS with only $10 \%$ spherical-shaped erythrocytes combined with jaundice, and splenomegaly. Moreover, consistent with the genetic findings, the anemia was ameliorated after splenectomy. This is the first report of $A N K 1$ c.4276C>T (p.R1426*) heterozygous nonsense mutation responsible for HS. Our results also demonstrate that NGS may provide a powerful approach for rapid genetic test of HS.

\section{MATERIALS AND METHODS}

\section{Subjects}

The male patient showed anemia and jaundice half a year after birth (hemoglobin: $95 \mathrm{~g} / \mathrm{L}$ ), was diagnosed with HS at 5 years old with $4.3 \%$ spherical-shaped erythrocytes on the peripheral blood smear and received blood transfusion for several time. At 8 years old, the genetic test upon the patient and his immediate family members was performed three months before a splenectomy due to anemia and splenomegaly with $10 \%$ sphericalshaped erythrocytes on the peripheral blood smear. The patient's father was 40 years old, and his mother was 32 years old. One month after the splenectomy, his hemoglobin and RBCs levels improved to normal range dramatically. Written informed consent was obtained from all participants. This study was formally approved by the Ethics Committee of Tongji Hospital, Tongji Medical College, Huazhong University of Science and Technology. All procedures were performed in accordance with the approved guidelines.

\section{Library preparation and Ion-Torrent PGM sequencing}

Ion Torrent adapter-ligated libraries were built using Ion Ampliseq ${ }^{\mathrm{TM}}$ Library Kit 2.0 (Thermo Fisher Scientific). Briefly, $10 \mathrm{ng}$ genomic DNA from peripheral blood mononuclear cell (PBMC) quantitated by Qubit 2.0 (Invitrogen, Carlsbad, CA, USA) was used for multiplex PCR amplification in two primer-pools with 118 and 115 amplicons respectively. The PCR products of the two pools were mixed together, and ligated to barcodes and Ion Torrent adapters (Life Technologies), followed by purification with AMPure XP beads (Beckman Coulter, Brea, CA, USA) and quantification by Ion Library TaqMan ${ }^{\text {TM }}$ Quantitation Kit. Barcoded libraries of different samples were pooled in equal amount and used as template for emulsion PCR using the $\mathrm{PGM}^{\mathrm{TM}} \mathrm{Hi}-\mathrm{Q}^{\mathrm{TM}}$ OT2 Kit on the Ion One Touch 2 Instrument, followed by enrichment of Ion Sphere Particles (ISPs) on the ES Instrument. Finally, 850 flows sequencing was done on the Ion $316^{\text {TM }}$ Chip v2 using Ion PGM Hi-Q Sequencing Kit on the Ion Torrent Personal Genome Machine (PGM).

\section{Bioinformatic analysis}

Raw sequence data were processed to trim adapter sequences, align to the hg19 human reference genome (GRCh37), analyze coverage and call variants on the Ion Torrent Server using the Torrent Suite software version 4.4.2 and the Variant Caller plugin, with the default parameters suggested by the manufacturer. Variant analysis was performed with the Ion Reporter 5.0 
software to classify variants as single nucleotide variations (SNVs), multiple nucleotide variants (MNV), insertions or deletions (indels). All found variants and regions below $20 \times$ coverage were visually verified with the Integrative Genomics Viewer (IGV) v2.3.8 (Broad Institute). Variants were annotated according to the nomenclature recommended by the Human Genome Variation Society (HGVS, http://www.hgvs.org) and their classification was attributed according to the Red Cell Membrane Disorder Mutations Database (RBCmembrane, http://research. nhgri.nih.gov/RBCmembrane), ClinVar https://www. ncbi.nlm.nih.gov/clinvar/, 1000G (http://asia.ensembl. org/index.html), HGMD (http://www.hgmd.cf.ac.uk/ac/ index.php), ExAC (http://exac.broadinstitute.org/), EVS (http://evs.gs.washington.edu/EVS/), and InterVar (http:// wintervar.wglab.org/) databases [9]. To predict possible impact of non-synonymous variants in exons, in-silico function was predicted by several software, including SIFT (http://sift.jcvi.org/www/SIFT_BLink_submit. html), MutationTaster (http://www.mutationtaster.org/), MutationAssessor (http://mutationassessor.org), Provean http://provean.jcvi.org/index.php and PolyPhen-2 (http:// genetics.bwh.harvard.edu/pph2/) [18-21]. The whole workflow was summarized in Figure 3.

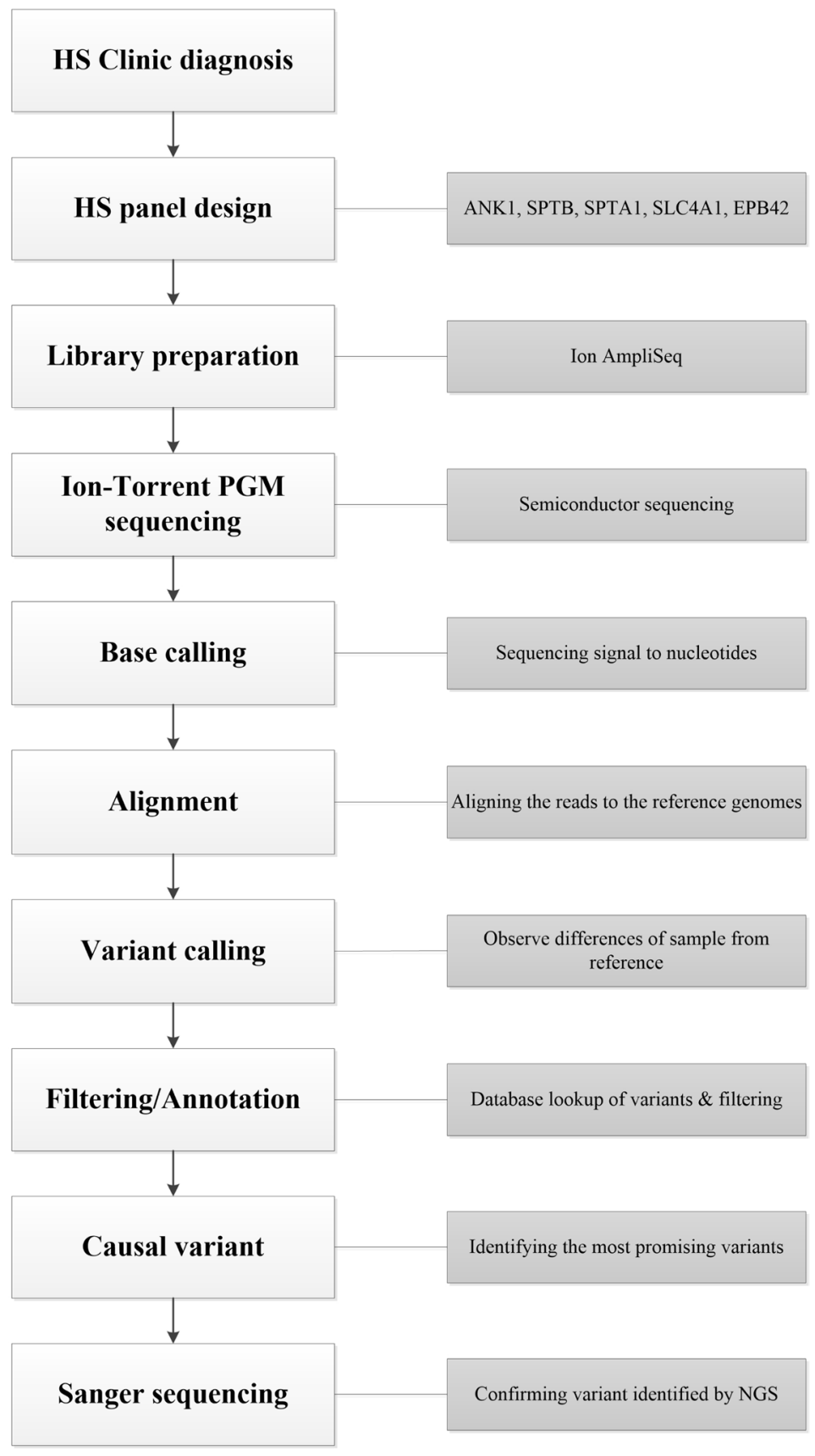

Figure 3: The workflow of this work. 
Table 3: Primer sequences used in this study

\begin{tabular}{cccc}
\hline Primer name & Forward & Reverse & Target \\
\hline ANK1 CD4 & GTCATAGAGAACAGGCTGGATC & GGCTTAGGTCCAAAACAATTC & rs1872877 \\
ANK1 CD9 & CTGGTGCTTGAGGAAACG & AAGTTTGCCGACCCTGAT & rs7826127 \\
ANK1 CD27 & CACCGAGACCTCAGACAACA & TCTCAGGGCTATGGACACC & rs504574 \\
ANK1 CD34 & GTCTGGCCATGCCTGTAAAG & AGGGTCCACAGCGTGAATG & rs2278621 \\
ANK1 CD35 & CTTCACTCCCACTCCTCCC & CTGCAAGATCAGGGGAAGAC & c.4276C $>$ T \\
\hline
\end{tabular}

\section{Sanger sequencing}

Sanger sequencing was performed as previously described [22]. Breifly, genomic DNA was extracted from PBMC using QIAamp DNA blood mini kit (QIAGEN, Hilden, Germany). Coding exons and splice junctions of ANK1, EPB42, SLC4A1, and SPTB genes (GenBank accession no. NM_001142446.1, NM_000119.2, NM_000342.3, NM_003126.2, and NM_001024858.2, respectively) were amplified for identified mutation, failed amplicons, or missed target region for amplicon design. Sanger sequencing of PCR products was performed bidirectionally on ABI 3500 Dx Genetic Analyzer (Applied Biosystems). Sequence was blasted on NCBI.

\section{Paternity testing and haplotype analysis}

Four single nucleotide polymorphisms (SNPs; rs2278621, rs504574, rs7826127, and rs1872877) ranging from chr8: 41690142 to chr8: 41728053 were selected from the UCSC Genome Browser (http://genome.ucsc. edu/), and linkage-disequilibrium analysis was performed based on LD TAG SNP selection (TagSNP; http://snpinfo. niehs.nih.gov/snpinfo/snptag.php). All primer sequences were listed in Table 3.

\section{CONFLICTS OF INTEREST}

None.

\section{FUNDING}

This work was supported by the National Natural Science Foundation of China (No. 81500925).

\section{REFERENCES}

1. Perrotta S, Gallagher PG, Mohandas N. Hereditary spherocytosis. Lancet. 2008; 372:1411-26. https://doi. org/10.1016/S0140-6736(08)61588-3.

2. Wang C, Cui Y, Li Y, Liu X, Han J. A systematic review of hereditary spherocytosis reported in Chinese biomedical journals from 1978 to 2013 and estimation of the prevalence of the disease using a disease model. Intractable Rare Dis Res. 2015; 4:76-81. https://doi.org/10.5582/ irdr.2015.01002.
3. Agre P, Orringer EP, Bennett V. Deficient red-cell spectrin in severe, recessively inherited spherocytosis. N Engl J Med. 1982; 306:1155-61. https://doi.org/10.1056/ NEJM198205133061906.

4. Gallagher PG. Hematologically important mutations: ankyrin variants in hereditary spherocytosis. Blood Cells Mol Dis. 2005; 35:345-47. https://doi.org/10.1016/j. bcmd.2005.08.008.

5. Platt OS, Lux SE, Falcone JF. A highly conserved region of human erythrocyte ankyrin contains the capacity to bind spectrin. J Biol Chem. 1993; 268:24421-26.

6. Rubtsov AM, Lopina OD. Ankyrins. FEBS Lett. 2000; 482:1-5. https://doi.org/10.1016/S0014-5793(00)01924-4.

7. Delaunay J. The molecular basis of hereditary red cell membrane disorders. Blood Rev. 2007; 21:1-20. https:// doi.org/10.1016/j.blre.2006.03.005.

8. Koboldt DC, Steinberg KM, Larson DE, Wilson RK, Mardis ER. The next-generation sequencing revolution and its impact on genomics. Cell. 2013; 155:27-38. https://doi. org/10.1016/j.cell.2013.09.006.

9. Li Q, Wang K. InterVar: Clinical interpretation of genetic variants by the 2015 ACMG-AMP guidelines. Am J Hum Genet. 2017; 100:267-80. https://doi.org/10.1016/j. ajhg.2017.01.004.

10. An X, Mohandas N. Disorders of red cell membrane. Br J Haematol. 2008; 141:367-75.

11. Miraglia del Giudice E, Nobili B, Francese M, D’Urso L, Iolascon A, Eber S, Perrotta S. Clinical and molecular evaluation of non-dominant hereditary spherocytosis. Br J Haematol. 2001; 112:42-47. https://doi.org/10.1046/j.13652141.2001.02501.x.

12. Hall TG, Bennett V. Regulatory domains of erythrocyte ankyrin. J Biol Chem. 1987; 262:10537-45.

13. Han JH, Kim S, Jang H, Kim SW, Lee MG, Koh H, Lee JH. Identification of a novel p.Q1772X ANK1 mutation in a Korean family with hereditary spherocytosis. PLoS One. 2015; 10:e0131251. https://doi.org/10.1371/journal. pone. 0131251 .

14. Hughes MR, Anderson N, Maltby S, Wong J, Berberovic Z, Birkenmeier CS, Haddon DJ, Garcha K, Flenniken A, Osborne LR, Adamson SL, Rossant J, Peters LL, et al. A novel ENU-generated truncation mutation lacking the spectrin-binding and C-terminal regulatory domains of Ank1 models severe hemolytic hereditary spherocytosis. Exp Hematol. 2011; 39:305-20, 20 e1-2. https://doi. org/10.1016/j.exphem.2010.12.009. 
15. Mouro-Chanteloup I, Delaunay J, Gane P, Nicolas V, Johansen M, Brown EJ, Peters LL, Van Kim CL, Cartron JP, Colin Y. Evidence that the red cell skeleton protein 4.2 interacts with the $\mathrm{Rh}$ membrane complex member CD47. Blood. 2003; 101:338-44. https://doi.org/10.1182/ blood-2002-04-1285.

16. Lu JT, Campeau PM, Lee BH. Genotype-phenotype correlation-promiscuity in the era of next-generation sequencing. N Engl J Med. 2014; 371:593-96. https://doi. org/10.1056/NEJMp1400788.

17. Agarwal AM, Nussenzveig RH, Reading NS, Patel JL, Sangle N, Salama ME, Prchal JT, Perkins SL, Yaish HM, Christensen RD. Clinical utility of next-generation sequencing in the diagnosis of hereditary haemolytic anaemias. Br J Haematol. 2016; 174:806-14. https://doi. org/10.1111/bjh.14131.

18. Kumar P, Henikoff S, Ng PC. Predicting the effects of coding non-synonymous variants on protein function using the SIFT algorithm. Nat Protoc. 2009; 4:1073-81. https:// doi.org/10.1038/nprot.2009.86.
19. Schwarz JM, Cooper DN, Schuelke M, Seelow D. MutationTaster2: mutation prediction for the deepsequencing age. Nat Methods. 2014; 11:361-62. https://doi. org/10.1038/nmeth.2890.

20. Choi Y, Chan AP. PROVEAN web server: a tool to predict the functional effect of amino acid substitutions and indels. Bioinformatics. 2015; 31:2745-47. https://doi.org/10.1093/ bioinformatics/btv195.

21. Adzhubei I, Jordan DM, Sunyaev SR. UNIT 7.20 Predicting Functional Effect of Human Missense Mutations Using PolyPhen-2. Curr Protoc Hum Genet. 2013; 76:7.20.17.20.41. https://doi.org/10.1002/0471142905.hg0720s76.

22. Wang X, Zhu Y, Shen N, Peng J, Wang C, Liu H, Lu Y. Mutation analysis of a Chinese family with oculocutaneous albinism. Oncotarget. 2016; 7:84981-88. https://doi. org/10.18632/oncotarget.13109. 